\title{
MORPHOLOGICAL TAGGING AND LEMMATIZATION IN THE ALBANIAN LANGUAGE
}

\author{
Diellza Nagavci Mati \\ Faculty of Contemporary Sciences and Technologies, South East European \\ University, Tetovo, Republic of North Macedonia \\ dn16574@seeu.edu.mk \\ Mentor Hamiti \\ Faculty of Contemporary Sciences and Technologies, South East European \\ University, Tetovo, Republic of North Macedonia \\ m.hamiti@seeu.edu.mk \\ Elissa Mollakuqe \\ Faculty of Information Sciences and Computer Engineering, \\ University Ss. Cyril and Methodius, Skopje, Republic of North Macedonia \\ elissamollakuqe@gmail.com
}

\begin{abstract}
An important element of Natural Language Processing is parts of speech tagging. With fine-grained word-class annotations, the word forms in a text can be enhanced and can also be used in downstream processes, such as dependency parsing. The improved search options that tagged data offers also greatly benefit linguists and lexicographers. Natural language processing research is becoming increasingly popular and important as unsupervised learning methods are developed. There are some aspects of the Albanian language that make the creation of a part-of-speech tag set challenging.

This research provides a discussion of those issues linguistic phenomena and presents a proposal for a part-of-speech tag set that can adequately represent them.
\end{abstract}


The corpus contains more than 250,000 tokens, each annotated with a medium-sized tag set. The Albanian language's syntagmatic aspects are adequately represented. Additionally, in this paper are morphologically and part-of-speech tagged corpora for the Albanian language, as well as lemmatize and neural morphological tagger trained on these corpora. Based on the held-out evaluation set, the model achieves $93.65 \%$ accuracy on part-of-speech tagging, The morphological tagging rate was $85.31 \%$ and the lemmatization rate was $88.95 \%$. Furthermore, the TF-IDF technique weighs terms and with the scores are highlighted words that have additional information for the Albanian corpus.

Key words: Part of speech tagging, Albanian language, Natural Language Processing, Lemmatization, Corpora.

\section{INTRODUCTION}

Linguistic data are necessary for many applications to facilitate communication, or at least to build linguistic datasets for use in natural language processing. About 7 million native Albanian speakers live in Albania, Kosovo, North Macedonia, and other Balkan countries. The Albanian language has complex grammar, which makes it an interesting and unique language to study This paradigm makes morphological tagging and lemmatization particularly challenging. Annotations, lemmatization tools, morphological analysis tools, and part-ofspeech tagging are not widely accessible for the language. In this paper, we aim to create a corpus of manually annotated part-of-speech tags, morphological features, and lemmas. As the name implies, part-of-speech tagging focuses on taking a text as an input and producing an output text where every word has an associated grammatical category such as an adjective, noun, verb, number, pronoun, etc.

According to this marking system, the meaning of a word and its adverbs are considered. The words in a grammatical category all have the same grammatical properties. Language identification began by taking advantage of the fact that short words have different frequencies in different languages (Pagliardini, Gupta, \& Jaggi, 2018). These were immediately followed by approaches that focused on $\mathrm{N}$-grams (sequences of $\mathrm{n}$ characters in a text).

Using Natural Language Processing, the text is collected into a corpus. Multilingual corpora may consist of texts that span one language or many languages. The purpose for which they are useful is numerous. Several attempts have been made to build a corpus for NLP tasks 
in Albanian (Mati, Ajdari, Raufi, Hamiti, \& Selimi, 2019). However, their small corpus sizes, different formats, and lack of public availability are significant drawbacks.

Several attempts have been made to develop NLP tools for Albanian. The following are some of the most important topics work-related to it in detail. Existing systems are often based on rules or dictionaries and are not available for online purposes. Although there is prior manually tagged corpora, none of them are publicly accessible.

Hasanaj presents two sets of tags: a small set of 16 tags and a large set of 326 tags. Besides the ten tags for parts of speech, there are three tags for delimiters, two tags for special cases (short forms of pronouns), and one tag for articles. In addition to encoding the major word classes, the large tagset also encodes additional features such as cases and numbers. (Hasanaj, 2012) investigates the two tagsets by utilizing maximum entropy and perceptron tagging. As contrasted to his gold standard corpora, his has only 263 tokens for the basic tagset and 641 for the large tagset.

A simple morphological analyzer is presented by Trommer et al. (2004), for the standard Albanian language. All the inflection types of Albanian language are covered by 340 sets of morphological rules. This tagset contains 17 labels adapted from the EAGLE guidelines description of the Albanian language. Based on an evaluation of 1,000 words (tokens), the morphological analyzer reaches oracle precision of $97 \%$ and recall of $92-95 \%$.

Another proposal for part-of-speech tagging is proposed by Kadriu (2013) who uses a lexicon of around 32,000 entries and their corresponding part-of-speech information. To deal with unknown words, she implements a stemming algorithm on top of the NLTK unigrams and regular expressions taggers. In this case, she analyzed 30 news articles across three domains. The paper does not mention the number of gold standard tokens.

Kabashi et al. (2016) presented a medium-sized tag set for Albanian, which consists of 67 tags and aims to adequately represent its morphosyntactic characteristics. Particularly, proposed articles and particles are treated in a linguistically sensible manner when combined with other word classes.

Our research aims to develop a morphological tagger as the main component of a comprehensive part-of-speech tagger for standardizing the Albanian language by constructing an annotated corpus for it. At the end of this paper, we have attempted to address this problem by implementing an unsupervised method by utilizing a tokenizer to split words into chunks and then calculating the frequency. Inflectional paradigms are especially rich in Albanian, making it particularly challenging to tag. So, there could be as many as 100 forms of a verb. 
Additionally, there are differences in inflectional patterns for the same syntactic categories: verbs are conjugated in 53 ways (Kote, Biba, Kanerva, Rönnqvist, \& Ginter, 2019), whereas noun stems are assigned plural affixes without any systematic basis. In addition, morphological tags indicate specific properties of words, such as part of speech, case tense, etc., for all word tokens in a text. It is also necessary to have a morphological disambiguation step, which selects from each tag set a unique tag for each token, as shown in figure $1^{1}$.

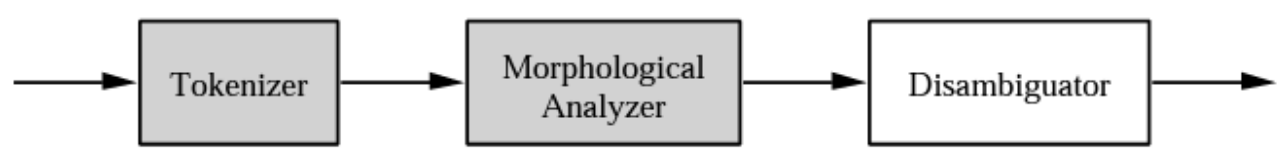

Figure 1. Architecture of PoS Tagger

\section{NATURAL LANGUAGE PROCESSING}

The goal of this field is to understand and produce natural language at several levels, including syntax, semantics, pragmatics, and dialog. A language has four levels: syntax, structure, semantics, and pragmatics. Natural Language Processing involves several steps, including tokenization, normalization, stemming, and part-of-speech tagging. As well as grammatical rules, NLP includes other approaches, such as inflection, derivation, tenses, semantic analyses, lexicon, and morphemes (Kurani \& Muho, 2014). All these approaches and rules were applied to the domain-based corpora for the Albanian language.

\section{Tokenization}

Natural Language Processing includes several steps, including tokenization. Tokenizing long strings is the process by which they are split into smaller parts or chunks. Hundreds of words can be tokenized from large sections of text. In addition, the process is generally completed once a piece of text has been appropriately tokenized (Mati, Ajdari, Raufi, Hamiti, \& Selimi, 2019). Since the Albanian alphabet contains letters such as 'ç' and '*' in text files, encoding the text files in UTF-8 can be a challenge during tokenization. It would be impossible to tokenize the files otherwise. Figure $2^{2}$ illustrates the tokenization process.

\footnotetext{
${ }^{1}$ Taken from: http://home.uni-leipzig.de/jtrommer/papers/tagger.pdf

${ }^{2}$ Taken from: https://rb.gy/exhdqf
} 


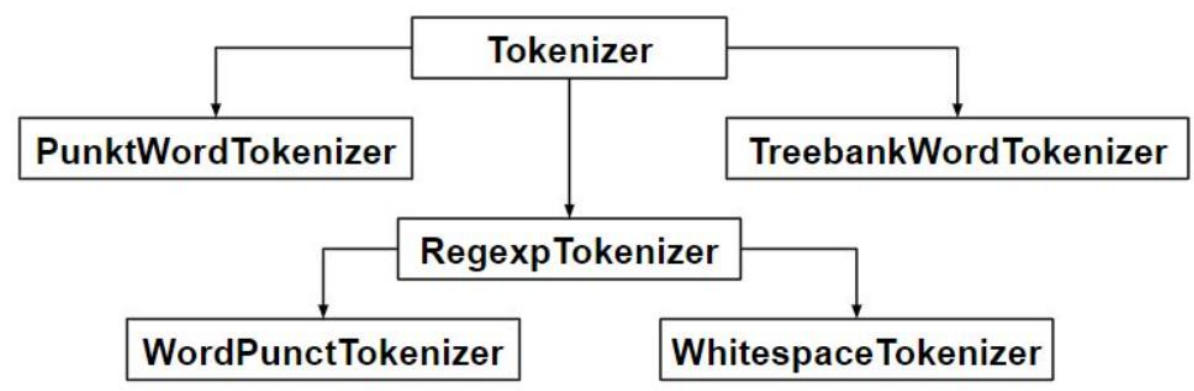

Figure 2. Tokenization

\section{Stemming and Lemmatization}

Lemmatization, as used in Natural Language Processing, refers to the process of removing suffixes, prefixes, infixes, circumfixes, and circumflexes from words and phrases so that they can be further processed (Kote, Biba, Kanerva, Rönnqvist, \& Ginter, 2019). Figure 3 shows examples of lemmatization in Albanian. The same basic functions are performed by stemming and lemmatizing. In stemming and lemmatizing, they reduce a word variant to its stem and lemma, respectively. Each concept differs slightly from the other. When stemming, a word is created by applying a set of rules, but the part of speech (POS) or context of the word is ignored. Similar to lemmatizing, lemmatizing involves reducing word forms to their root form after understanding the POS and the context of the given word (Balakrishnan \& LloydYemoh, 2014).

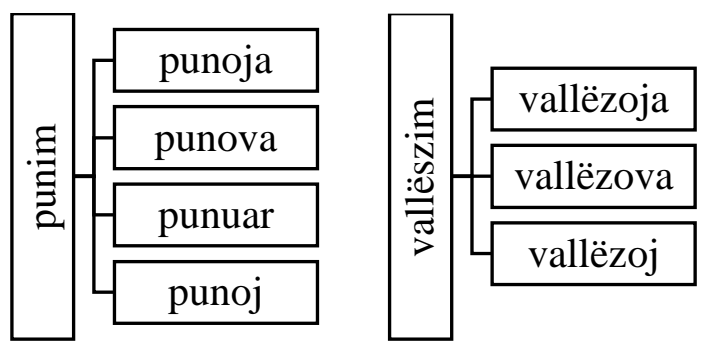

Figure 3. Stemming examples in the Albanian language

\section{Corpus}

Natural Language Processing describes the collection of text as a corpus. There are numerous reasons why multilingual corpora may be useful, whether they contain texts in one language or span many. The development of a corpus for NLP tasks in Albanian has been attempted numerous times. Despite this, they suffer from significant drawbacks, such as small corpus sizes, various formats, and, moreover, it is not possible to access them online. The paper 
describes the creation of a corpus of 250k Albanian sentences drawn from texts of various fields in electronic sources.

\section{MORPHOLOGY TAGGING OF THE ALBANIAN LANGUAGE}

More than 7 million Albanian speakers make up this language branch of IndoEuropean. A close relationship between these languages is unknown. Among Albanian nouns, there are eight cases (nominative, accusative, genitive, dative, ablative, and vocative) and two numbers (singular and plural) (Trommer, The Post-Syntactic Morphology of the Albanian PrePosed Article: Evidence for Distributed, 2000).

Adjectives: There are five different groups of adjectives in the Albanian language based on the suffixes -e (feminine gender), -a (feminine plural), -e (masculine plural), or zero marking in various partially overlapping distributions (Toska, Nivre, \& Zeman, 2020). Adjectives have the morphological categories of gender, number, and case dependent on related nouns. In addition, adjectives have a degree of gradation. There are three types of grades: positive, comparative, and superlative. The adjective is graded by adding comparative particles, or adverbs, to the base word. In the case of comparative gradation, the particle "më" is used, e.g., positive: " $\mathrm{i}$ vogël" (engl. small), comparative: "më i vogël” (engl. smaller) and superlative: "më i vogli" (engl. the smallest). The adjective can be placed before or after the verb It can be preceded by an article or not and describes a noun. According to this proposal, there are five categories for adjectives in Albanian, including those that occur before nouns, those with proposed articles, and those which do not inflect. The table 1 below shows the results.

Table 1. Descriptions of Albanian adjectives tags

\begin{tabular}{|c|l|l|l|}
\hline$\#$ & Tag & Name & Example in Albanian language \\
\hline 1. & Adj & Adjective & djali trim \\
\hline 2. & AdjA & Adj.precededbyarticle & ndërtesë e lartë \\
\hline 3. & AdjP & Preposedadj. & trimi djalë \\
\hline 4. & AdjPA & Prep.adj.prec.byart. & e mira vajzë \\
\hline 5. & AdjN & Noninflected adjective & Blu/neto \\
\hline
\end{tabular}

Nouns: In addition to a variety of inflections for nouns (singular, plural), there are numerous case forms (nominative, dative, accusative, ablative), such as: houses PL-ABL-DEF, (engl. of houses) (Piton \& Lagji, 2010). In contrast to definiteness and case marking, which 
are quite predictable based on phonology, stem gender and number, the plural suffix (- e, Ø, e, or-a) is rather unpredictable. The Albanian language noun tags are listed in Table 2. Consider the following example: 'Të rinjtë janë nga Kosova.', Eng. 'The young people are from Kosovo.', which is analyzed as Të\Art rinjtëlNArt janëlV nga\Prep KosovalNm.IPunct. (Nagavci Mati, Hamiti, Susuri, Selimi, \& Ajdari, 2021)

\begin{tabular}{|c|l|l|l|}
\hline \multicolumn{3}{|c|}{ Table 2. Descriptions of Albanian noun tags } \\
\hline$\#$ & Tag & Name & Example in Albanian language \\
\hline 1. & N & Noun & mërkurë \\
\hline 2. & NA & Noun preceded by article & e mërkurë \\
\hline 3. & NHg & Het. noun & kuq(sg.m.) vs.kuqe(pl.f.) \\
\hline
\end{tabular}

Verbs: Verb inflections in Albanian are the most complex. Besides three different tenses (present, aorist, imperfect), there are also two different voices (active and passive), and five different moods (indicative, subjunctive, optative, imperative, and admirative). Phonology is partly responsible for allomorphy in verbal inflection. Furthermore, thus verbs ending in vowels form the 1 st person aorist with -va (e.g. vizato-va, 'I drew') while stems ending in consonants take-a (e.g. hap-a, 'I opened') (Rushali \& Kiwelekar, 2020). In traditional Albanian grammars, verbs are divided into different inflectional classes, which results partly in a variety of allomorphs, and a genitive case is also assumed in order to combine with the dative. In addition to these synthetic tenses, there are two analytic tenses: future (formed with the present subjunctive and the particle do and the perfect form with the participle form and nite forms of the auxiliaries kam, 'have', and jam, 'be'. affixes (e.g., for $1 \mathrm{sg}-\mathrm{j}$ in mëso-j, 'I learn' and -m in the-m, 'I say') (Kabashi \& Proisl, 2016). Table 3 below shows the proposed verb tags for the Albanian language.

Table 3. Albanian language proposed verb tags

\begin{tabular}{|c|l|l|l|}
\hline$\#$ & Tag & Name & Example in Albanian language \\
\hline 1. & V & Verb (finite forms)) & Tha \\
\hline 2. & VPart & Participle(non-finiteforms) & Thënë \\
\hline 3. & VCl & V.w.clitic & i tha \\
\hline 4. & VImpv & Imperativeform & Prit / Fol \\
\hline 5. & VImpvCl & Imperativew.clitic & Shpjegomëni \\
\hline 6. & VPass & V.w.pass.part.u & U bë \\
\hline & & & \\
\hline
\end{tabular}




\begin{tabular}{|c|l|l|l|}
\hline 7. & VPassCl & V.w.pass.part.andclitic & Ua tha \\
\hline 8. & VSubj & V.w.subj.particle & Të thotë \\
\hline 9. & VSubjCl & V.w.pass.part.andcl. & Ta tha \\
\hline 10. & VSubjPass & V.w.subj.a.pass.part & T'u thotë \\
\hline 11. & VAux & Auxiliar verb & kam \\
\hline 12. & VMod & Modal verb & mund \\
\hline 13. & VRecp & Reciprocal verb & njihen \\
\hline 14. & VRef & Reflexive verb & ushqehem \\
\hline
\end{tabular}

Numerals: In the Albanian language are divided into cardinal and ordinal numbers. There are no differences morphologically between the ordinal numbers. Gradation is always presented as an adjective, except in categories. Using this as an example, we could say: 'Kjo ishte ndeshja e saj e dytë brenda një muaji.' - Eng. 'This was her second match within one month.', which is tagged as Sot\Adv ishtelV ndeshjalN e\Art dytëlNumO e\Art saj\PossPr brenda\Prep një\NumC muajilNo.IPunc. Table 4 below shows the proposed numeral tags for the Albanian language.

Table 4. Albanian language proposed numeral tags

\begin{tabular}{|r|l|l|l|}
\hline$\#$ & Tag & Name & Example in Albanian language \\
\hline 15. & NumC & Cardinal number & dy lojë \\
\hline 16. & NumO & Ordinal number & loja e dytë \\
\hline
\end{tabular}

Pronoun: There are seven types of pronouns according to their meaning and sense:

- Possessive Pronouns includes: i im, e, tu, i, saj, etc.

- Interrogative Pronouns: kush, cili, cila, etc.

- Pronouns that are demonstrative: ky, kjo, ai, ajo, etc.

- Subject Pronouns: unë, ti, ai, ajo, ne, ju, ata, ato.

- Pronouns used as relative pronouns are: që, i cili, e cila, të cilat, etc.

- Indefinite Pronouns: dikush, askush, ndonjë, etc.

- Reflexive Pronouns: veten, vetveten. 
The subjective pronouns, "ai" and "ajo" can also be demonstrative pronouns if they are followed by a noun. Articles can precede some pronouns, like nouns and adjectives. Additionally, there is a short form of subjective pronouns.

\section{EXPERIMENTAL AND RESULTS}

We have obtained our results by implementing the corpus in Albanian using the Natural Language Toolkit. There are several open-source program modules for linguistic purposes in NLTK. NLTK provides both symbolic and statistical approaches to natural language processing and interfaces them to annotated corpora. The NLTK Python modules provide data types, tasks, simple corpora, and problem sets associated with Natural Language Processing. The corpus contains 631,008 words. After eliminating duplicates, words with characters such as numbers, hyphens, apostrophes, and roman numerals, the unique words amount to 49,514 words. Using the Albanian language corpus, Table 5 illustrates frequency and part of speech examples. In Albanian language corpus, the word 'Angli' occurs at a frequency of $0.100 \%$ as noun in part-od-speech, whereas the word 'kërkues' is an adjective as part-of-speech with a frequency of $0.100 \%$. "Afërmi" and "ngrohtë" are two adverbs in Albanian that occur with a frequency of approximately $0.0001 \%$. As injections, we have the words 'ore' and 'ose' with a frequency of $0.00001 \%$. Prepositions with ablative can be found in the words 'përgjatë' and 'rreth' with a $0.00001 \%$ frequency.

Table 5. POS examples from the Albanian corpus

\begin{tabular}{|c|l|l|l|}
\hline$\#$ & Word & Frequency & Part of Speech \\
\hline 1. & Angli & $0.100 \%$ & Noun \\
\hline 2. & fillore & $0.200 \%$ & Noun \\
\hline 3. & kërkues & $0.100 \%$ & Adjective \\
\hline & & & \\
\hline 4. & zemërmadh & $0.0001 \%$ & Adjective \\
\hline 5. & ngatërroj & $0.0001 \%$ & Verb \\
\hline 6. & prezentoj & $0.0001 \%$ & Verb \\
\hline & & & \\
\hline 7. & afërmi & $0.00001 \%$ & Adverb \\
\hline 8. & ngrohtë & $0.00001 \%$ & Adverb \\
\hline 9. & ore & $0.000001 \%$ & Interjection \\
\hline & & & \\
\hline
\end{tabular}




\begin{tabular}{|c|l|l|l|}
\hline \multicolumn{1}{|l|}{} & & & \\
\hline 10. & ose & $0.00001 \%$ & Interjection \\
\hline 11. & përgjatë & $0.00001 \%$ & Preposition with ablative \\
\hline 12. & rreth & $0.00001 \%$ & Preposition with ablative \\
\hline
\end{tabular}

The accuracy of token and sentence segmentation, part-of-speech tagging, and morphological analysis is measured Lemmatization and features.

Table 6. An evaluation of the tasks of morphological tagging, text segmentation, and lemmatization of Albanian.

\begin{tabular}{|l|l|}
\hline Target & Accuracy \% \\
\hline Token & $97.85 \%$ \\
\hline Sentences & $97.92 \%$ \\
\hline Part of Speech & $93.65 \%$ \\
\hline Lemmas & $88.95 \%$ \\
\hline Features & $85.31 \%$ \\
\hline
\end{tabular}

The TF-IDF model is commonly used for information retrieval. Using these word occurrences, the vector models can be created without relying on any precise ordering. We have considered two approaches in the preprocessing phase:

1) TF (Term Frequency) and

2) TF-IDF (“Term Frequency, Inverse Document Frequency).

An important word (term) in a document is rated based on how frequently it appears in different documents. The IDF (inverse document frequency) calculates how much information is in a word. Calculated by dividing the total number of documents $\mathrm{N}$ by the number of documents containing the term $\mathrm{i}$.

TF-IDF is calculated using the following formula (1):

$$
w_{i, j}=t f_{i, j} \times \log \left(\frac{N}{d f i}\right)
$$

TF-IDF for a term $\mathrm{i}$ in a document $\mathrm{j}$ :

- $\mathrm{t} f_{\mathrm{i}, \mathrm{j}}=$ number of occurrences of $\mathrm{i}$ in $\mathrm{j}$,

- $\mathrm{d} f_{\mathrm{i}}=$ number of documents containing I, 
- $\mathrm{N}=$ total number of documents.

By using TfidfVectorizer, it is possible to tokenize documents, learn the vocabulary and inverse document frequency weightings, and encode new documents. The following table illustrates the use of TfidfVectorizer which helps to learn vocabulary and inverse document frequencies across three small documents from the Albanian language and then encode one of those documents.

Table 7. Results for the first experiment.

\begin{tabular}{|l|l|l|l|}
\hline TF-IDF & Index & S1 & S2 \\
\hline gjuha & 2 & 1.6931 & 0.6388 \\
\hline zyrtare & 11 & 1.6931 & 0.0000 \\
\hline në & 3 & 1.6931 & 0.4261 \\
\hline republikën & 5 & 1.0000 & 0.2517 \\
\hline shqipërisë & 8 & 1.6931 & 0.0000 \\
\hline është & 13 & 1.2876 & 0.3241 \\
\hline shqipja & 6 & 1.6931 & 0.4261 \\
\hline shteti & 9 & 1.6931 & 0.0000 \\
\hline asnjanës & 0 & 1.6931 & 0.4261 \\
\hline çështjet & 12 & 1.6931 & 0.00000 \\
\hline besimit & 1 & 1.6931 & 0.00000 \\
\hline sistemi & 10 & 1.6931 & 0.42618 \\
\hline qeverisjes & 7 & 1.6931 & 0.00000 \\
\hline shqipëris & 4 & 1.2876 & 0.32412 \\
\hline & & & \\
\hline
\end{tabular}

A vocabulary of 14 words is learned from the documents and each word is assigned a unique integer index in the output vector represented by table 7 . The inverse document frequencies are calculated for all words in the vocabulary, with "është" at index 13 receiving the lowest score of 0 . Furthermore, the first document is encoded as a sparse array of 14 elements, and we can examine the final scores for each word according to different scores for "gjuha", "shteti," and "sistemi." from the other words in the vocabulary. 


\section{CONCLUSION}

This paper presents one of the largest electronically tagged and morphologically analyzed corpus of the Albanian language containing more than 250,000 tokens. The evaluation experiments show a promising outcome including the possibility of expanding the dictionary. The annotations are reviewed manually and comply with the universal dependencies morphological tag sets. By training this pipeline on this corpus, we have achieved promising results in terms of tagging and lemmatization. Increasing the number of sources is expected to further improve the tagger's accuracy since we intend to train relatively good models on a larger corpus in order to allow fully automatic tagging. 


\section{REFERENCES}

1. Balakrishnan, V., \& Lloyd-Yemoh, E. (2014). Stemming and Lemmatization: A Comparison of Retrieval Performances. Lecture Notes on Software Engineering, 262267.

2. Hasanaj, B. (2012). A Part of Speech Tagging Model for Albanian. Saarbr"ucken: LAP Lambert Academic Publishing.

3. Kabashi, B., \& Proisl, T. (2016). A proposal for a part-of-speech tagset for the Albanian language. In Proceedings of the Tenth International Conference on Language Resources and Evaluation (LREC'16) (pp. 4305-4310). Portorož, Slovenia: The International Conference on Language Resources and Evaluation.

4. Kadriu, A. (2013). NLTK tagger for Albanian using iteraIterative Approach. Proceedings of the 35th Internationa Conference on Information Technology Interfaces (ITI).

5. Kote, N., Biba, M., Kanerva, J., Rönnqvist, S., \& Ginter, F. (2019). Morphological Tagging and Lemmatization of Albanian: A Manually Annotated Corpus and Neural Models. Computation and Language (cs. CL), 50-62.

6. Kurani, A., \& Muho, A. (2014). A morphological comparative study between Albanian and English language. European Scientific Journal (European Scientific Institute). England.

7. Mati, D. N., Ajdari, J., Raufi, B., Hamiti, M., \& Selimi, B. (2019). A Systematic Mapping Study of Language Features Identification from Large Text Collection. 2019 8th Mediterranean Conference on Embedded Computing (MECO). Budva, Montenegro, Montenegro: IEEE.

8. Nagavci Mati, D., Hamiti, M., Susuri, A., Selimi, B., \& Ajdari, J. (2021, July 1st). Building Dictionaries for Low Resource Languages: Challenges of Unsupervised Learning. Annals of Emerging Technologies in Computing (AETiC), 52-58,. doi:0.33166/AETiC.2021.03.005

9. Pagliardini, M., Gupta, P., \& Jaggi, M. (2018). Unsupervised Learning of Sentence Embeddings using Compositional n-Gram Features. Conference of the North American Chapter of the Association for Computational Linguistics (pp. 528-540). North American: IEEE. doi:10.18653/v1/N18-1049

10. Piton, O., \& Lagji, K. (2010). Morphological study of Albanian words, and processing with NooJ. Computation and Language, (pp. 189-205). Barcelona.

11. Rushali , D., \& Kiwelekar, A. (2020). Deep Learning Techniques for Part of Speech Tagging by Natural Language Processing. 2020 2nd International Conference on Innovative Mechanisms for Industry Applications (ICIMIA). 2, pp. 50-62. Bangalore, India: IEEE. doi:10.1109/ICIMIA48430.2020.9074941

12. Toska, M., Nivre, J., \& Zeman, D. (2020). Universal Dependencies for Albanian. Proceedings of the Fourth Workshop on Universal Dependencies, (pp. 178-188). Barcelona. 
13. Trommer, J. (2000). The Post-Syntactic Morphology of the Albanian Pre-Posed Article: Evidence for Distributed. Proceedings of the third conference on South-Slavic and Balkan languages, Plovdiv (pp. 8-16). IEEE.

14. Trommer, J., \& Kallulli, D. (2004). A Morphological Tagger for Standard Albanian. In Proceedings of the Fourth International Conference on Language Resources and Evaluation (LREC'04)., (pp. 1-8). Vienna. 